\title{
Design and Evaluation of a Wearable Haptic Device for Skin Stretch, Pressure, and Vibrotactile Stimuli
}

\author{
M. Aggravi*, F. Pausé*, P. Robuffo Giordano, C. Pacchierotti
}

\begin{abstract}
This paper presents a wearable haptic device for the forearm and its application in robotic teleoperation. The device is able to provide skin stretch, pressure, and vibrotactile stimuli. Two servo motors, housed in a 3D printed lightweight platform, actuate an elastic fabric belt, wrapped around the arm. When the two servo motors rotate in opposite directions, the belt is tightened (or loosened), thereby compressing (or decompressing) the arm. On the other hand, when the two motors rotate in the same direction, the belt applies a shear force to the arm skin. Moreover, the belt houses four vibrotactile motors, positioned evenly around the arm at 90 degrees from each other. The device weights $220 \mathrm{~g}$ for $115 \times 122 \times 50 \mathrm{~mm}$ of dimensions, making it wearable and unobtrusive. We carried out a perceptual characterization of the device as well as two human-subjects teleoperation experiments in a virtual environment, employing a total of 34 subjects. In the first experiment, participants were asked to control the motion of a robotic manipulator for grasping an object; in the second experiment, participants were asked to teleoperate the motion of a quadrotor fleet along a given path. In both scenarios, the wearable haptic device provided feedback information about the status of the slave $\operatorname{robot}(s)$ and of the given task. Results showed the effectiveness of the proposed device. Performance on completion time, length trajectory, and perceived effectiveness when using the wearable device improved of $19.8 \%$, $25.1 \%$, and $149.1 \%$ than when wearing no device, respectively. Finally, all subjects but three preferred the conditions including wearable haptics.
\end{abstract}

Index Terms-Haptics and Haptic Interfaces, Wearable Robots, Telerobotics and Teleoperation.

\section{INTRODUCTION}

W EARABLE haptic devices have shown promising results in the fields of robotic teleoperation [1], [2], rehabilitation [3], guidance [4], [5], and gaming [6], [7]. They can be easily worn by the human user and are able to provide effective, rich, localized, and private stimuli. Moreover, wearable interfaces can also easily enable the engaging of multicontact interactions and operations [8]. Finally, ungrounded haptic devices have also been proven to guarantee the stability and safety of haptic-enabled teleoperation loops [1], [9].

As per the location of the wearable device, the forearm has been often chosen as an effective place for providing haptic

Manuscript received: September, 8, 2017; Revised December, 21, 2017; Accepted February, 11, 2018.

This paper was recommended for publication by Editor Yasuyoshi Yokokohji upon evaluation of the Associate Editor and Reviewers' comments. This research has received funding from Rennes Métropole under the "Allocation d'Installation Scientifique (AIS) 2017" programme and from the European Union H2020 RoMaNS project 645582.

* These authors contributed equally to the manuscript.

M. Aggravi, F. Pausé, P. Robuffo Giordano, and C. Pacchierotti are with CNRS, Univ Rennes, Inria, IRISA in Rennes, France. \{marco.aggravi, prg, claudio.pacchierotti\}eirisa.fr, florent.pauseeinria.fr.

Digital Object Identifier (DOI): see top of this page.

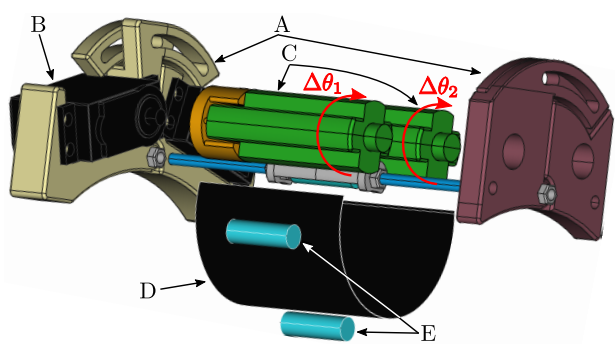

(a) CAD design.

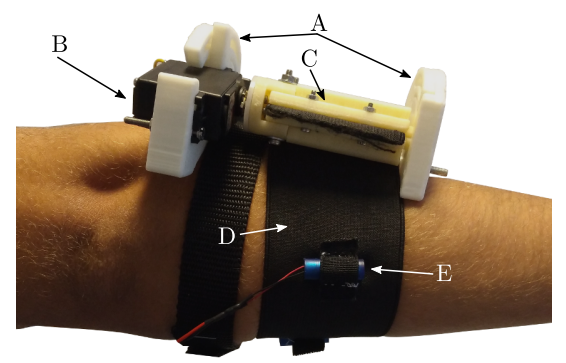

(b) Device worn on the forearm.

Fig. 1. The proposed wearable device for the arm. It consists of a static platform (A) that accommodates two servomotors (B) and two pulleys (C), a fabric belt (D), and four vibrotactile motors (E). The device is able to provide skin stretch, pressure, and vibrotactile stimuli to the arm.

guidance and feedback in various robotics-related applications, such as robotic teleoperation and human-robot interaction. For example, Bark et al. [10] presented a wearable haptic feedback device able to impart rotational skin stretch to the hairy skin of the forearm. Subjects were able to use feedback from the device to control the positioning of a virtual object within \pm 6.5 degrees of the total range of motion. Stanley and Kuchenbecker [11] evaluated ten different forms of tactile feedback for wrist rotation guidance. The devices are capable of tapping, dragging across, squeezing, twisting, or vibrating against the user's wrist. Results showed that directional responses are fastest when direction is conveyed through the location of the tactile stimulus or steady lateral skin stretch. More recently, Casini et al. [12], [13] developed a wearable device, called CUFF, for the haptic stimulation of the user's arm with pressure and stretch cues. It is composed of two DC motors attached to a fabric cuff worn around the arm. Scheggi et al. [4] presented a cooperative human-robot navigation system in which vibrotactile armbands provide the human wearer with information about the motion of a mobile robot, keeping the two agents in a soft formation. Chinello et al. [14] used four pulleys, worn evenly around the arm, to provide skin stretch at the palmar, dorsal, ulnar, and radial sides of the arm.

Within this context, this paper discusses a novel wearable 
haptic device and its evaluation in robotic teleoperation. The proposed device is able to provide cutaneous haptic feedback to the forearm through pressure, vibrotactile, and skin stretch haptic feedback, as shown in Fig. 1. The device consists of two servo motors moving an elastic belt placed in contact with the user's arm skin. Similarly to [12], when the motors spin in opposite directions, the belt tightens or loosens around the user's arm, while when the motors spin in the same direction, the belt applies a shear force to the skin. Moreover, it also houses four vibrotactile motors, positioned evenly around the arm at 90 degrees from each other. The device weights $220 \mathrm{~g}$ for $115 \times 122 \times 50 \mathrm{~mm}$ of dimensions. Combining these three types of haptic stimuli (pressure, skin stretch, vibrotactile) in such a compact package, the device can provide a wide set of feedback information to the human wearer in a comfortable and unobtrusive way [15]. We present the design and characterization of the device, as well as the results of two human-subject teleoperation experiments in a virtual environment. In the first experiment, participants were asked to control the motion of a simulated 6-degrees-of-freedom (6-DoF) robotic manipulator for grasping an object; in the second experiment, participants were asked to teleoperate the motion of a fleet of ten simulated drones along a given path. In both scenarios, the proposed haptic interface provided feedback information about the status of the slave robot(s) and of the given task.

\section{THE HAPTIC DEVICE}

A prototype of our device is shown in Fig. 1b, while Fig. 1a indicates its main parts. Referring to Fig. 1a, the device consists of an ABS static platform (A) that houses two HS-625MG servo motors (B) and two pulleys (C), a 6-cm-wide elastic fabric belt (D) that applies the requested stimuli to the skin, and four 307-100 Pico Vibe vibrotactile motors (E) positioned evenly around the arm at 90 degrees from each other. A strap band is used for securing the device on the forearm (see Fig. 1b).

The cutaneous device can provide pressure and skin stretch stimuli by moving the fabric band. When the two servo motors rotate in opposite directions, the belt is tightened or loosened, providing a force normal to the arm skin. On the other hand, when motors spin in the same direction, the belt applies a shear force to the arm skin. The servomotors are position controlled, which means that it is only possible to command a desired angle. The relationship between the commanded angle and belt displacement for each motor is $\Delta b_{c, i}=r \Delta \theta_{i}, i=1,2$, being $r=12.2 \mathrm{~mm}$ the radius of the servo motor pulley, $\Delta b_{c, i}$ the commanded belt displacement due to the motion of motor $i$, and $\Delta \theta_{i}$ the $i$-th motor commanded angle, expressed in radians (see Fig. 1a). In our case, the two motors always rotate of the same amount, i.e., $\left|\Delta \theta_{1}\right|=\left|\Delta \theta_{2}\right|$ and $\left|\Delta b_{c, 1}\right|=\left|\Delta b_{c, 2}\right|$. As introduced above, the motion of the two motors results in either a belt lateral displacement $\Delta b_{l, c}$ (if the motors rotate in the same direction) or in a tightening of the belt $\Delta b_{p, c}$ (if the motors rotate in opposite directions),

$$
\left\{\begin{array}{lll}
\Delta b_{l, c}=\operatorname{sgn}\left(\Delta \theta_{2}\right) \Delta b_{c, 2} & \text { if } & \operatorname{sgn}\left(\Delta \theta_{1}\right)=\operatorname{sgn}\left(\Delta \theta_{2}\right) \\
\Delta b_{p, c}=2 \operatorname{sgn}\left(\Delta \theta_{2}\right) \Delta b_{c, 2} & \text { if } & \operatorname{sgn}\left(\Delta \theta_{1}\right) \neq \operatorname{sgn}\left(\Delta \theta_{2}\right) .
\end{array}\right.
$$

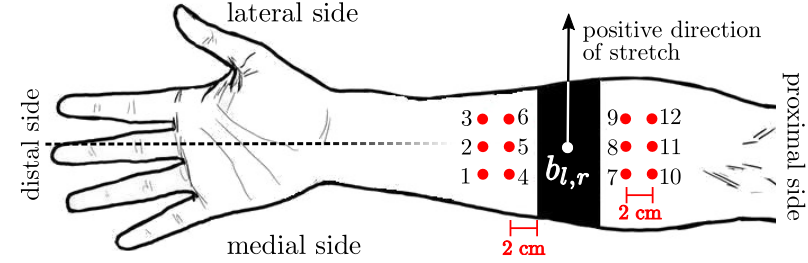

Fig. 2. Device characterization. Commanding different stretches to the belt, we analyzed the real belt displacement w.r.t. the stretch imparted on the arm skin around the belt. We considered 12 points on the arm and one of the belt.

A positive value for $\Delta b_{l, c}$ indicates a stretch toward the lateral side of the arm, while a negative value indicates a stretch toward the medial side (see Fig. 2). Similarly, a positive value for $\Delta b_{p, c}$ indicates a tightening of the belt, while a negative value indicates a loosening of the belt. In addition to this type of stimuli, the device can also provide vibrotactile feedback at four equidistant points in the arm, allowing the system to easily convey directional information (Top, Left, Down, and Right w.r.t. the cross section of the arm). Each vibrating motor $m \in\{T, L, D, R\}$ has an effective vibration frequency range $f_{m} \in[0-280] \mathrm{Hz}$ and a square-wave-like vibration pattern $\beta_{m}$, i.e., when motor $m$ is activated, it vibrates at frequency $f_{m}$ for a period of $2 \beta_{m}$ with duty cycle $50 \%$.

\section{DEVICE CHARACTERIZATION}

To understand how to correctly modulate the device motor inputs, we evaluated the (i) belt shearing effect on the skin when moving the servo motors in the same direction, the (ii) normal force applied on the skin when moving the servo motors in opposite directions, and the correspondent (iii) differential thresholds of perception. Moreover, we used the characterization of [16] to effectively modulate the vibromotors inputs for guidance. These four mappings will be used in Sec. IV for controlling the device in the two experiments.

\section{A. Skin stretch stimuli}

This Section analyzes the real belt lateral displacement $\Delta b_{l, r}$ resulting from a commanded belt lateral displacement $\Delta b_{l, c}$ (see (1)). In fact, although using an elastic fabric band guarantees high comfort and ease of use, it introduces uncertainties in the actuation system, i.e., $\Delta b_{l, c}$ may differ from $\Delta b_{l, r}$. A human user (male, 22 years old, wrist $\varnothing 5.4 \mathrm{~cm}$, arm $\varnothing 9.2 \mathrm{~cm}$ ) was asked to wear our device on his right forearm, as shown in Fig. 2. The device laid on a table, with the belt facing up. The user was asked to keep his arm still during the experiment. Twelve black dots were drawn on his forearm, six between the device and the wrist and six between the device and the elbow. Each block of six dots was arranged in a $3 \times 2$ array, with a dot-to-dot distance of $2 \mathrm{~cm}$. The belt was then commanded to move clockwise and counterclockwise around the arm, $\Delta b_{l, c}=$ $[-19.2,-12.8,-8.5,-4.3,4.3,8.5,12.8,19.2] \mathrm{mm}$, which means rotating both motors of $[-90,-60,-40,-20,20,40$, $60,90]$ degrees (see (1)). After each stretch stimulus, the experimenter took a picture of the setup, in order to measure the real displacement of the belt $\Delta b_{l, r}$ and the stretch of the 


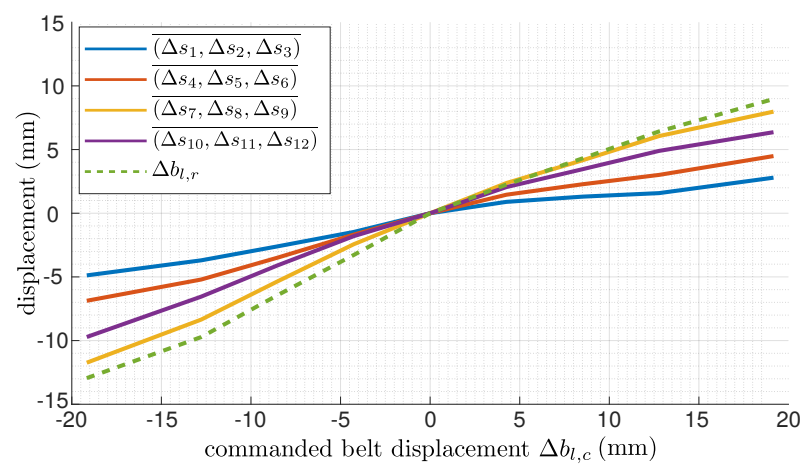

Fig. 3. Skin stretch characterization. It shows the real belt displacement $\Delta b_{l, r}$ and skin stretch $\Delta s$ resulting from a commanded belt displacement $\Delta b_{l, c}$. To make the graph easier to read, we aggregated the data coming for neighboring points on the skin, showing their mean displacement three by three.

skin around the belt $\Delta s=\left[\Delta s_{1}, \ldots, \Delta s_{12}\right]^{T}$. The device was well tighten to the arm for preventing any slippage of the belt w.r.t. the skin. Figure 3 shows the commanded belt displacement $\Delta b_{l, c}$ vs. the real belt displacement $\Delta b_{l, r}$ and the stretch of the twelve points on the skin $\Delta s$. It is interesting to notice that proximal points follow more closely the motion of the belt. Moreover, medial commanded belt displacements (negative values in Fig. 3) result in larger skin stretches and in larger actual belt displacements with respect to lateral (positive) commanded belt displacements.

In the experiments of Sec. IV, to better secure the device on the forearm, we use a second stationary belt, which fixes the upper static platform to the proximal side of the arm (see Fig. 1b). While this solution is very simple and effective, it prevents part of the forearm from stretching properly (i.e., the area around points 10-12 in Fig. 2). As indicated in Sec. V, in the future, we will study different approaches for fixing the device onto the forearm, with the objective of eliciting the most effective sensations.

\section{B. Pressure stimuli}

This Section analyzes the applied squeezing action $\varphi_{p}$ given a commanded tightening of the belt $\Delta b_{p, c}$. The same human user as in Sec. III-A was asked to wear again our cutaneous device on his right forearm, as in Fig. 2. A FSR 402 force sensor was placed on the belt, opposite to the static platform housing the motors and in contact with the volar skin of the arm (near point $b_{l, r}$ in Fig. 2). The cutaneous device was then commanded to tighten the belt around the arm, shortening it every $1.5 \mathrm{~mm}$ from 0 to $76.5 \mathrm{~mm}$, i.e., $\Delta b_{p, c}=[0,1.5,3, \ldots, 76.5] \mathrm{mm}$. After each squeeze stimulus, the experimenter saved the force $\varphi_{p}$ applied by the belt on the arm. The belt was kept in place for $5 \mathrm{~s}$ and the sensor values averaged. The starting force applied to the arm, i.e., when $\Delta b_{p, c}=0$, was $2.5 \mathrm{~N}$. This procedure was repeated three times and the resulting data averaged. Figure 4 shows the commanded belt squeeze $\Delta b_{p, c}$ vs. the average squeezing force $\varphi_{p}$.

\section{Differential thresholds}

The differential threshold, often referred to as JND, gives us information about how different two stimuli generated by our device need to be in order to be perceived as different

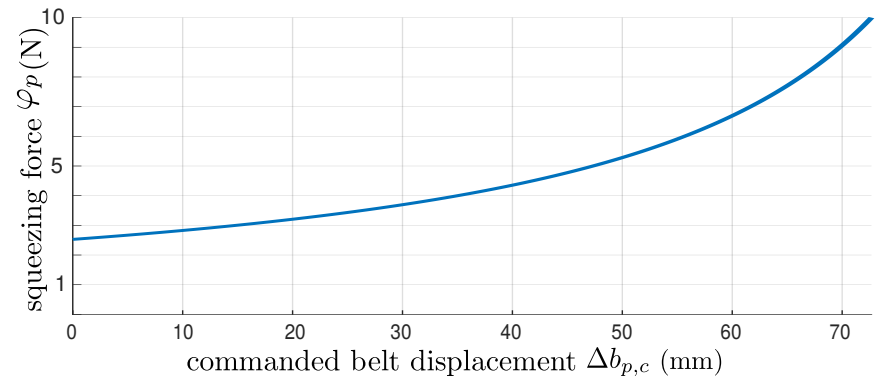

Fig. 4. Pressure characterization. Commanding different displacements $\Delta b_{p, c}$ of the belt, we analyzed the force applied by the device on the volar skin of the user's arm.

by a human user. Ten participants took part in the experiment (two women and eight men). We evaluated the differential thresholds using the method of constant stimuli, both for the skin stretch and for the squeezing stimuli. This method involves the presentation of different stimulus levels (e.g., skin stretch) in random order. Unlike the methods of limits, this approach employs a nonsequential procedure: a step size and range of intensities is selected which, based upon previous experience, include the target threshold level. The subject's task is to say whether two stimuli are the different or the same. We tested the stretch JND at six standard stimuli, $\Delta b_{l, c}=[-10.5,-6.3,-2.1,2.1,6.1,10.5] \mathrm{mm}$, with a stepsize of $0.1 \Delta b_{l, c}$ between the considered standard stimulus $\Delta b_{l, c}$ and the maximum tested value $1.5 \Delta b_{l, c}$. Similarly, we tested the squeeze JND at four standard stimuli, $\Delta b_{p, c}=$ $[7.7,23.0,38.3,53.7] \mathrm{mm}$, with a step-size of $0.1 \Delta b_{p, c}$ between the considered standard stimulus and the maximum tested value $1.5 \Delta b_{p, c}$.

Subjects were required to wear the cutaneous device as shown in Fig. $1 \mathrm{~b}$ and tell the experimenter when the two provided stimuli felt different. Each participant performed 18 trials of the squeezing stimuli procedure, with four repetitions for each reference stimulus, and 12 trials of the skin stretch stimuli procedure, with two repetitions for each reference stimulus. The average JND was calculated by finding individual JNDs and then averaging them together. The device showed a differential threshold at the $75 \%$ level of $33.2 \%$ and $24.7 \%$ of the standard stimulus for the skin stretch and squeeze, respectively, which is in agreement with previous results in the literature [14], [17].

\section{EXPERIMENTAL EVALUATION}

\section{A. Experiment \#1: shared-control of a robotic telemanipulator}

1) Experimental setup: this first evaluation considers a shared-control teleoperation experiment in a virtual environment. It is inspired by [18], that describes the algorithmic design of a shared-control architecture for visual-based teleoperation. In [18], a grounded kinesthetic interface has been used for providing force cues, but no - even minor - evaluation was presented. The experimental setup is shown in Fig. 5. The master side consists of the human operator, wearing the proposed cutaneous device, and a Leap Motion controller in charge of tracking the position and orientation of the user's hand. The slave side consists of a 6-DoF Viper S850 robotic arm carrying a gripper, simulated using the popular 


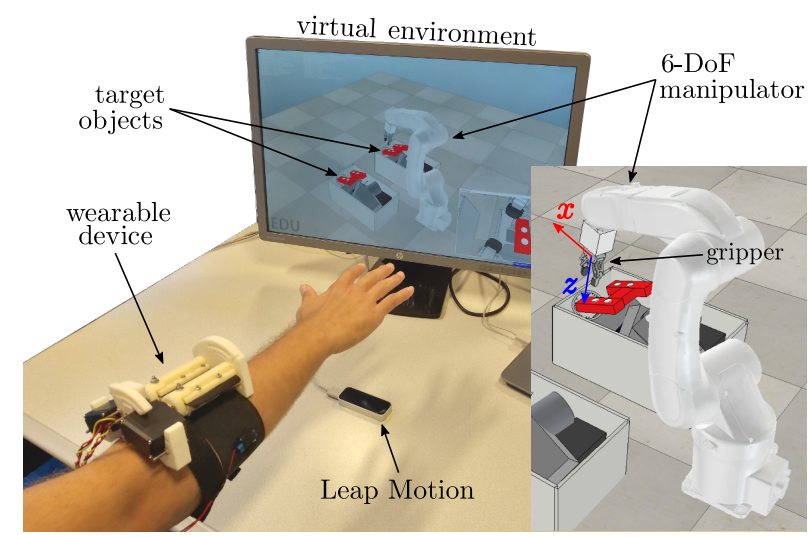

Fig. 5. Experiment \#1. The human operator wears the wearable cutaneous device on his right arm, and a Leap Motion controller tracks the position and the orientation of his hand. The slave side is composed of a simulated 6-DOF Viper S850 robotic arm carrying a gripper, and two objects to grasp.

V-REP environment, and two objects to grasp. The autonomous algorithm proposed in [18] is in charge of always keeping the manipulator gripper pointed towards the object to be grasped (2 DoF) with the aim of assisting the human operator in the grasping task. At the same time, the human operator is given the possibility to steer the slave end-effector along the remaining 4 null-space directions (w.r.t. the main pointing task) using the Leap Motion controller.

2) Methods: As explained, the autonomous algorithm controls 2 DoF of the robotic manipulator by keeping the gripper always oriented towards the object to be grasped. The gripper is therefore constrained to move on the surface of a virtual sphere centered at the object. The human operator can instead actuate the remaining $4 \mathrm{DoF}$ in the null-space of the autonomous pointing task, that is, moving the gripper across the surface of the sphere, toward/away from the object (i.e., changing the radius of the sphere), and rotating the gripper along the pointing direction $z$ towards the object (see Fig. 5). The haptic cues introduced in [18] are meant to inform the operator about how close the slave manipulator is to given constraints (singularities and joint limits in the considered scenario). These constraints are encoded in a global cost function $H(\boldsymbol{q})$, with $\boldsymbol{q} \in \mathbb{R}^{6}$ being the manipulator joint configuration vector. The force cues $\tau \in \mathbb{R}^{4}$, displayed on the $4 \mathrm{DoF}$ actuated by the operator, are then obtained by projecting the joint motion caused by the $i$-th user command $(i=1, \ldots, 4)$ onto the negative gradient of $H(\boldsymbol{q})$ : in this way, the operator is informed about the direction (in the space of the user commands) along which $H(\boldsymbol{q})$ would diminish or, equivalently, along which she/he would move away from the constraints (again, we refer to [18] for details). The operator can then easily react to the provided cues by adjusting the commanded motion in order to keep away from the constraints, an action that would be, otherwise, hard to infer and implement by simply looking at the slave manipulator.

In our case, we used the wearable device for providing the operator with the above-mentioned force cues $\tau=\left[\tau_{1}, \tau_{2}, \tau_{3}\right.$, $\left.\tau_{4}\right]^{T} \in \mathbb{R}^{4}$, being $\tau_{4}$ the torque cue corresponding to the rotation of the gripper along the direction towards the object, and $\tau_{l}=\left[\tau_{1}, \tau_{2}, \tau_{3}\right]^{T}$ the remaining three cues corresponding to translations of the gripper along its $x, y, z$ axes, respectively.
In particular, we chose to display $\tau_{l}$ by means of vibrotactile cues, while $\tau_{4}$ was provided through skin stretch stimuli. This choice is motivated by successful past experiences in providing similar information. Aggravi et al. [16] used four vibrating motors at the wrist to guide an operator along a 3D path, while Chinello et al. [19] used skin stretch at the wrist to convey rotation guidance. In what follows, we details how such haptic cues are obtained. Each component of $\tau$ is processed so that it is zeroed if lower, in absolute value, than a threshold $t h_{i}^{\tau}=$ $[0.01 \mathrm{~N}, 0.01 \mathrm{~N}, 0.01 \mathrm{~N}, 0.1745 \mathrm{Nm}]$, and it is constrained between maximal values $\mathrm{T}_{i}=[15 \mathrm{~N}, 15 \mathrm{~N}, 15 \mathrm{~N}, 2 / \pi \mathrm{Nm}]$, that is, with $i=1, \ldots, 4$,

$$
\tau_{i}^{*}=\left\{\begin{array}{lll}
0 & \text { if } & \left|\tau_{i}\right| \leq t h_{i}^{\tau} \\
\max \left(\min \left(\tau_{i} / \mathrm{T}_{i}, 1\right),-1\right) & \text { if } \quad\left|\tau_{i}\right|>t h_{i}^{\tau}
\end{array} .\right.
$$

With such regularized values for $\tau$, we compute the frequencies for the vibrotactile motors and the angular values for the servo motors. For the former, we need to map six possible directions (left, right, top, down, backward, and forward) to the four available motors (Top, Right, Down, and Left, see Sec. II). Similarly to [16], we directly map the left $L$ and right $R$ motors to the force cues on the $y$-axis of the gripper (see Fig. 5), that is

$$
\left\{\begin{array}{lll}
f_{R}=0, f_{L}=-280 \tau_{2}^{*} & \text { if } & \operatorname{sgn}\left(\tau_{2}\right)<0 \\
f_{L}=0, f_{R}=280 \tau_{2}^{*} & \text { if } & \operatorname{sgn}\left(\tau_{2}\right)>0
\end{array} .\right.
$$

Then, we use the top motor $T$ to tell the user to both "go up" and "go forward", and the down motor $D$ to tell the user to both "go down" and "go backward" [16]. Of course, motors $T$ and $D$ need different vibration patterns for the two different directions they convey, i.e., "go up" should have a different pattern from "go forward", and "go down" should have a different vibration pattern from "go backward". This is achieved by changing the interval between each vibration. In particular, $T$ will vibrate in bursts of $\beta_{T}=100 \mathrm{~ms}$ for "go forward" and of $\beta_{T}=200 \mathrm{~ms}$ for "go up". Similarly, $D$ will vibrate in bursts of $\beta_{D}=100 \mathrm{~ms}$ for "go backward" and of $\beta_{D}=200 \mathrm{~ms}$ for "go down". To avoid sudden and possibly confusing changes between up/forward or down/backward stimuli, we weight the forward/backward feedback $\tau_{1}$ more than the top/down feedback $\tau_{3}$ by multiplying the former of $\delta=1.5$. Finally, to obtain vibration inputs $f_{T}, f_{D}, \beta_{T}$, and $\beta_{D}$, we compare $\tau_{1}$ and $\tau_{3}$ as follows

$$
\begin{aligned}
& \left\{\begin{array}{rl}
f_{T}=280 \tau_{1}^{*}, \beta_{T}=100 \mathrm{~ms} & \text { if } \tau_{3}=0 \text { and } \delta \tau_{1}>t h_{1}^{\tau} \text { or } \\
\tau_{3} & \neq 0 \text { and } \delta \tau_{1} \geq\left|\tau_{3}\right| \\
f_{T}=-280 \tau_{3}^{*}, \beta_{T}=200 \mathrm{~ms} & \text { if } \tau_{1}=0 \text { and } \tau_{3}<-t h_{1}^{\tau} \text { or } \\
\tau_{1} & \neq 0 \text { and } \tau_{3}<\left|\delta \tau_{1}\right|
\end{array},\right. \\
& \left\{\begin{aligned}
f_{D}=-280 \tau_{1}^{*}, \beta_{D}=100 \mathrm{~ms} & \text { if } \tau_{3}=0 \text { and } \delta \tau_{1}<-t h_{1}^{\tau} \text { or } \\
\tau_{3} & \neq 0 \text { and } \delta \tau_{1} \leq\left|\tau_{3}\right| \\
f_{D}=280 \tau_{3}^{*}, \beta_{D}=200 \mathrm{~ms} & \text { if } \tau_{1}=0 \text { and } \tau_{3}>t h_{1}^{\tau} \text { or } \\
\tau_{1} & \neq 0 \text { and } \tau_{3}>\left|\delta \tau_{1}\right|
\end{aligned}\right.
\end{aligned}
$$

As per the stretch, the commanded angle for each servo motor comes directly from $\tau_{4}^{*}$ as

$$
\Delta b_{l, c}=19.2 \tau_{4}^{*}
$$

The desired velocity for the robot end-effector at time $t$, $\boldsymbol{v}_{e e}(t)$, is obtained by tracking the user's hand with the Leap 


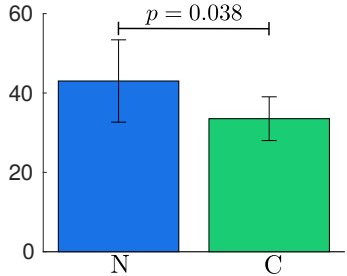

(a) Completion time (s)

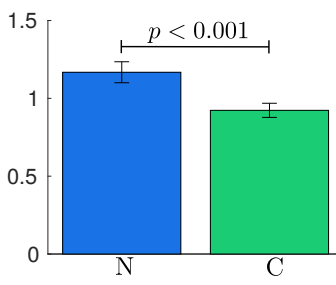

(b) Trajectory length $(\mathrm{m})$

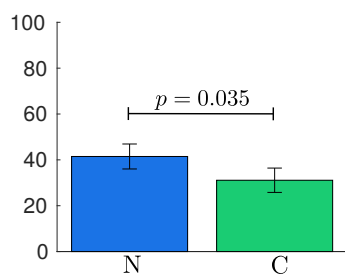

(c) Haptic actuation (\%)

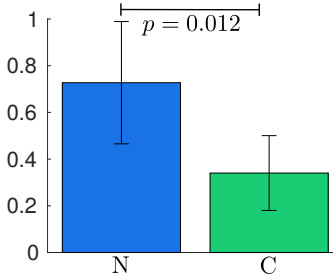

(d) $\tau_{4, R M S}^{*}(\mathrm{~N} / \mathrm{m})$

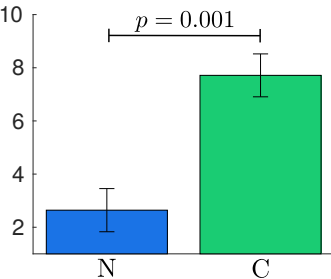

(e) Perceived effectiveness

Fig. 6. Experiment \#1. The task consisted of steering the robotic arm to grasp two target objects. Completion time, path followed by the robot, haptic actuation, gripper rotation error, and perceived effectiveness (with range from 1 to 9) provided a measure of performance. Mean and $95 \%$ confidence interval are plotted.

Motion in the following way. The keyboard works as a clutch and, when the space key is pressed, the current pose of the user's hand with respect to the Leap Motion base $\boldsymbol{H}(t) \in \mathrm{SE}(3)$ is saved, $\boldsymbol{H}(0)=\boldsymbol{H}(t)$, being $\mathrm{SE}(3)$ the special Euclidean group and $\boldsymbol{H}(t)$ composed of $\left[\mathbf{R}^{H}(t), \boldsymbol{p}^{H}(t)\right]$, where $\mathbf{R}^{H} \in$ $\mathrm{SO}(3)$ is the rotation matrix between the Leap Motion frame and the hand frame, and $\boldsymbol{p}^{H} \in \mathbb{R}^{3}$ is the position of the hand (see Fig. 5).

As the hand moves, the desired end-effector velocity is calculated from the position of the hand $\boldsymbol{p}^{H}(t)$ w.r.t. the initial value $\boldsymbol{p}^{H}(0)$ saved when the user started pressing the clutch,

$$
\boldsymbol{v}_{e e}(t)=\mathbf{B}_{e e}\left[\begin{array}{c}
\boldsymbol{p}^{H}(t)-\boldsymbol{p}^{H}(0) \\
\psi^{H}(t)-\psi^{H}(0)
\end{array}\right]
$$

where $\psi^{H}(t)$ is the yaw angle of the hand w.r.t. the Leap Motion frame, and $\mathbf{B}_{e e} \in \mathbb{R}^{4 \times 4}$ is a diagonal matrix of gains. This clutching approach allows the user to start the motion with the hand at an arbitrary position with respect to the Leap Motion, enabling him to pause, move to a more comfortable or suitable position, and then resume the control of the robot. The main advantage of this position-velocity control mode (also used in [18]) is that the operator is not limited by the workspace of the Leap Motion. The V-REP simulation is shown in a screen placed in front of the user.

The task consists of steering the robotic arm towards two objects to be grasped. Once the first object (placed on the right hand side of the manipulator) is reached, the second object becomes the target one, and the user is requested to steer the robotic arm towards it. The task started when the subject moved the robot for the very first time and finished when the subject reached the second object. As in [18], the grasping phase was not considered, and subjects were only requested to place the gripper close to the target object. However, subjects were also asked to orient the gripper such that it would be able to correctly grasp the objects. A video of the experiment is available as supplemental material.

Each participant made six trials of the grasping task, with three randomized repetitions for each feedback condition:

(C) cutaneous feedback provided by the wearable device,

(N) no haptic feedback.

In condition $\mathrm{C}$, the haptic feedback conveyed by our cutaneous device provides the human operator with information about the feasibility of the user's commands with respect to possible constraints of the robotic system, as detailed in (2)-(5). Pressure (squeeze) feedback is not used in this experiment. In condition $\mathrm{N}$, the human operator does not receive any haptic information. In both conditions, the subject is able to see the simulated environment on the screen. Trials were randomized to avoid any learning effect. Data from the same experimental condition and subject were averaged before the analysis [20].

3) Subjects: Fourteen participants took part in the experiment, including four women and ten men (age $21-31$ ). The experimenter explained the procedures and adjusted the setup to be comfortable for each user. One practice trial was allowed.

4) Results: In order to evaluate the effectiveness of our teleoperation system and the usefulness of wearable haptic feedback, we evaluated (i) the completion time, (ii) the robot path length, (iii) the amount of haptic stimuli provided during the task, and (iv) the perceived effectiveness of the different conditions. All data passed the Shapiro-Wilk normality test.

Figure 6a shows the average task completion time. A paired-samples t-test determined that the task completion time differed statistically significantly between conditions $(t(13)=2.312, p=0.038, a=0.05)$. Figure $6 \mathrm{~b}$ shows the average linear motion covered by the robotic gripper during the task. A paired-samples t-test determined that this average linear motion differed statistically significantly between conditions $(t(13)=6.952, p<0.001, a=0.05)$. Figure $6 \mathrm{c}$ shows the percentage of time the cutaneous device was activated, either providing vibrations or skin stretch. This metric measures how much the user deviates from the constrained trajectory defined by the shared-control algorithm. In condition $\mathrm{N}$, when users did not wear the cutaneous device, this metric indicates the percentage of time the cutaneous device would have been activated if worn. A paired-samples t-test determined that the percentage of time the cutaneous device was activated differed statistically significantly between conditions $(t(13)=2.358, p=0.035, a=0.05)$. Figure $6 \mathrm{~d}$ shows the root-mean-square of $\tau_{4}^{*}$, which indicates the error in following the gripper rotational cues during the task, provided through skin stretch feedback (see (5)). This metric assesses the benefits of the skin stretch guidance. A paired-samples t-test determined that this rotational error differed statistically significantly between conditions $(t(13)=2.899, p=0.012, a=0.05)$. Immediately after the experiment, subjects were also asked to report the effectiveness of both feedback conditions in completing the given task using bipolar Likert-type ninepoint scales. Fig. 6e shows the perceived effectiveness. A Wilcoxon signed rank test showed a statistically significant difference between the means of the two feedback conditions $(z=3.304, p=0.001, a=0.05)$. Finally, twelve out of fourteen subjects found using wearable haptic feedback to be the most effective at completing the grasping task. 


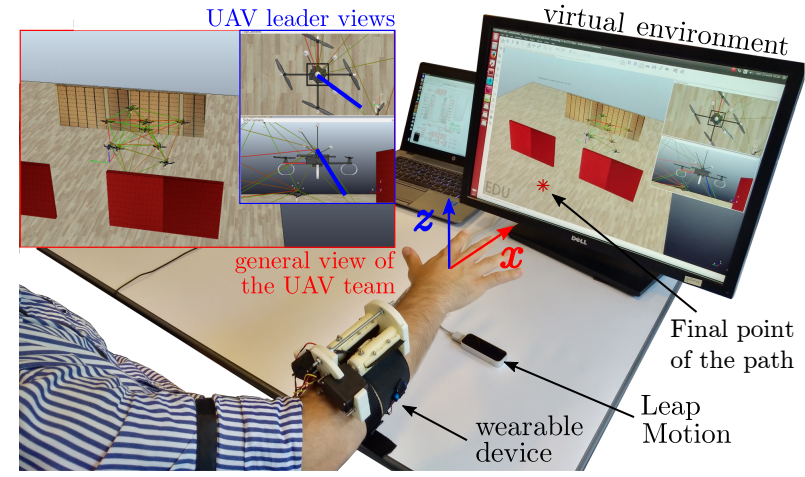

Fig. 7. Experiment \#2. The human operator wears the wearable cutaneous device, and a Leap Motion controller tracks the position of his hand. The slave side is composed of a fleet of ten quadrotors. One is controlled by the operator and the rest moves according to the connectivity-maintenance strategy of [21].

\section{B. Experiment \#2: teleoperation of Unmanned Aerial Vehicles}

1) Experimental setup: this second evaluation considers an experiment of teleoperation of a fleet of ten quadrotors, simulated again using V-REP. It is inspired by [21], [22], where it is presented the algorithmic design of a connectivitymaintenance strategy for telenavigating a group of quadrotors in a cluttered environment. However, no human-subject evaluation was presented. The connectivity-maintenance algorithm of [21], [22] considers sensing/communication constraints among the robot pairs (max. range and line-of-sight occlusions) as well as other requirements, such as inter-robot and obstacle avoidance, and formation control. By properly designing the weights on the edges of the multi-robot sensing/communication graph, it is possible to encode the mentioned constraints and requirements into a single scalar quantity $\lambda_{2}$, i.e., the second-smallest eigenvalue of the (state-dependent) Laplacian matrix associated to the interaction graph. In particular, the weights are designed so that if $\lambda_{2}=0$ the interaction graph is disconnected or a collision with an obstacle/robot has occurred, and $\lambda_{2}>0$ otherwise. The agents then implement a gradient-based action aimed at maintaining $\lambda_{2}(t)>0$ at all times despite the possible presence of additional motion commands (e.g., one or multiple quadrotors being steered by an external planner or a human operator). Finally, as explained in [22], the user can also set at runtime a minimum reference value $\lambda_{2}^{d}>0$ for $\lambda_{2}$, forcing the group to become more or less "connected" during motion by increasing or decreasing the minimum threshold $\lambda_{2}^{d}$.

In our proposed experimental setup, shown in Fig. 7, the human user commands only one robot inside the formation, the leader, by providing a desired velocity $3 \mathrm{D}$ vector. The other robots are then forced to follow the leader by the enforced connectivity-maintenance controller since, otherwise, the graph would eventually become disconnected as the leader navigates in the environment. As explained, the connectivity-maintenance controller enforces, at the same time, both inter-robot and obstacle collision avoidance. We also note that the leader motion is influenced by the operator (who is commanding its desired velocity), but also by the connectivity maintenance action which is representative of the whole group status (e.g., if a robot in the group is lagging behind because of an obstruction, the leader would be automatically slowed down and eventually stopped to avoid breaking the graph connectivity). Therefore, by mapping (via force cues) the leader's response to the human commands, the operator is informed about the whole group status/mobility. As in Sec. IV-A, the master side consists of the human operator, wearing the proposed cutaneous device, and a Leap Motion controller.

2) Methods: The desired velocity for the leader at time $t$, $\dot{\mathbf{q}}_{1}^{d}(t)$, is obtained using the same reasoning as in Sec. IV-A1. By clutching the user's hand position and giving the robot a velocity proportional to the distance between the current hand position $\boldsymbol{p}^{H}(t)$ and its initial value $\boldsymbol{p}^{H}(0)$, we have

$$
\dot{\mathbf{q}}_{1}^{d}(t)=\mathbf{B}_{q}\left(\boldsymbol{p}^{H}(t)-\boldsymbol{p}^{H}(0)\right),
$$

where $\mathbf{B}_{q} \in \mathbb{R}^{3 \times 3}$ is a diagonal matrix of gains. The closure of the hand, similarly to [2], is instead computed for setting the value of the connectivity threshold $\lambda_{2}^{d}$. The V-REP simulation of the environment is shown in a screen in front of the user.

The task consists in following a predefined 3D path, passing through $M=20$ via-points, $\mathcal{P}=\left\{\boldsymbol{x}_{1}, \ldots, \boldsymbol{x}_{M}\right\}, \boldsymbol{x}_{k} \in \mathbb{R}^{3}$. The position of these points is unknown to the user. After the second-last point $\boldsymbol{x}_{M-1}$, the target path force the leader - and possibly the entire formation - to pass through an aperture in the virtual scene (between the two red walls in Fig. 7). To do so, the user needs to compact the team by increasing the desired connectivity value $\lambda_{2}$ by closing her/his hand. We set this target $\lambda_{2}$ value to be $\lambda_{2 \text {,final }}=3$. A video of the experiment is available as supplemental material. Similarly to Sec. IV-A, we considered two feedback conditions, $\mathrm{V}$ and $\mathrm{CV}$.

In condition $\mathrm{V}$, information about the location of the next viapoint is provided by a blue segment spawning from the leader robot toward the via-point (see the leader views in Fig. 7). The length of the segment increases linearly with the distance between the leader and the via-point: $0 \mathrm{~m}$ when the leader is on the via-point and $1 \mathrm{~m}$ when the leader is farer than $1 \mathrm{~m}$ from the via-point. In addition to navigation information, when users are trying to increase the value of $\lambda_{2}$ by closing their hands, the difference between the desired and current values of $\lambda_{2}$ is shown on the screen. In condition $\mathrm{CV}$, we show again the blue segment pointing at the next via-point, as in condition V. However, we also provide guiding vibrotactile stimuli.

The considered guiding method is inspired by [16]. Let $\mathbf{q}_{1} \in \mathbb{R}^{3}$ be the 3D position of the leader robot and $\boldsymbol{x}^{*}$ the next via-point to reach. Let us define the distance between $\mathbf{q}_{1}$ and $\boldsymbol{x}^{*}$ as $\mathbf{d}_{q}=\boldsymbol{x}^{*}-\mathbf{q}_{1}$ and regularize it as in Sec. IV-A1,

$$
d_{q, i}= \begin{cases}0 & \text { if } \quad\left|d_{q, i}\right| \leq t h^{d} \\
\max \left(\min \left(d_{q, i} / D_{\max }, 1\right),-1\right) & \text { if } \begin{array}{l}
\left|d_{q, i}\right|>t h^{d} \\
i
\end{array} \\
i=\{1,2,3\},\end{cases}
$$

with maximal distance $D_{\max }=1.5 \mathrm{~m}$ and distance threshold $t h^{d}=0.2 \mathrm{~m}$. This threshold also determines the switching criterion between via-points: if the leader robot enters the 3D cube defined by $t h^{d}$, the new target via-point becomes the one that follows $\boldsymbol{x}^{*}$ in $\mathcal{P}$. When $\boldsymbol{x}^{*}=\boldsymbol{x}_{M}$ and $d_{q, i}<t h^{d}$, for any $i=\{1,2,3\}$, the task is completed. Although here we use vibrations to guide the leader robot along the predefined 3D path, we calculate the commanded vibromotor frequencies 


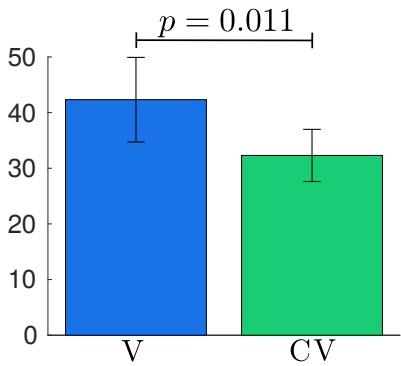

(a) Trajectory length $(\mathrm{m})$

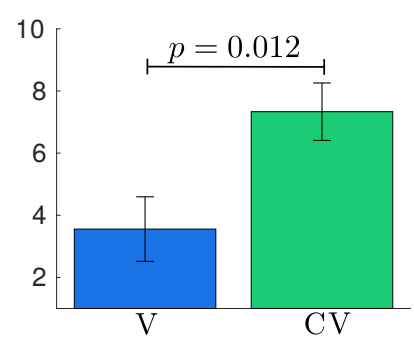

(b) Perceived effectiveness
Fig. 8. Experiment \#2. The task consisted of following a predetermined trajectory in the simulated space. Completion time, path followed by the robot, and perceived effectiveness (with range from 1 to 9) provided a measure of performance. Mean and 95\% confidence interval are plotted.

similarly to Sec. IV-A. Therefore, frequencies for motors left $L$ and right $R$ are

$$
\left\{\begin{array}{ll}
f_{L}=0, f_{R}=-280 d_{q, 2}^{*} & \text { if } \quad \operatorname{sgn}\left(d_{q, 2}\right)<0 \\
f_{R}=0, f_{L}=280 d_{q, 2}^{*} & \text { if } \quad \operatorname{sgn}\left(d_{q, 2}\right)>0
\end{array},\right.
$$

while frequencies for motors top $T$ and down $D$ are

$$
\begin{aligned}
& f_{T}=280 d_{q, 1}^{*}, \beta_{T}=100 \mathrm{~ms} \quad \text { if } d_{q, 3}=0 \text { and } \delta d_{q, 1}>t h^{d} \text { or }
\end{aligned}
$$

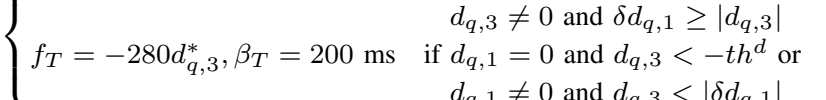

$$
\begin{aligned}
& \int f_{D}=-280 d_{q, 1}^{*}, \beta_{D}=100 \mathrm{~ms} \text { if } d_{q, 3}=0 \text { and } \delta d_{q, 1}<-t h^{d} \text { or } \\
& \left\{\begin{aligned}
d_{q, 3} & \neq 0 \text { and } \delta d_{q, 1} \leq\left|d_{q, 3}\right| \\
f_{D}=280 d_{q, 3}^{*}, \beta_{D}=200 \mathrm{~ms} \quad \text { if } d_{q, 1} & =0 \text { and } d_{q, 3}>t h^{d} \text { or } \\
d_{q, 1} & \neq 0 \text { and } d_{q, 3}>\left|\delta d_{q, 1}\right|
\end{aligned}\right.
\end{aligned}
$$

with $\delta=1.5$ (see Sec. IV-A for details).

In addition to navigation information, as the user tries to increase the value of $\lambda_{2}$ by closing her/his hand, the cutaneous device provides a squeeze action $\Delta b_{p, c}$, proportional to the difference $\hat{\lambda}_{2}=\lambda_{2}^{d}-\lambda_{2}$ between the desired and current value of $\lambda_{2}$. It is indeed important to gradually increase $\lambda_{2}^{d}$. In fact, increasing it suddenly may lead to aggressive maneuvers of the robots, which may in turn have a negative effect on the overall stability of the formation. The commanded/desired value for $\lambda_{2}$ is defined as $\lambda_{2}^{d}=3 o_{h}$, where $o_{h} \in[0-1]$ describes how much open the hand is ( $o_{h}=0$ with a hand fully open and $o_{h}=1$ with a hand closed in a fist [2]). This leads to a commanded squeeze stimulus of

$$
\Delta b_{p, c}= \begin{cases}0 & \text { if } \hat{\lambda}_{2}<t h_{\min }^{\lambda} \\ \frac{76.5}{2}\left(1-\cos \left(\alpha_{\lambda} \hat{\lambda}_{2}+\gamma_{\lambda}\right)\right) & \text { if } t h_{\min }^{\lambda} \leq \hat{\lambda}_{2}<t h_{\max }^{\lambda} \\ 76.5 & \text { if } \hat{\lambda}_{2} \geq t h_{\max }^{\lambda}\end{cases}
$$

with $t h_{\min }^{\lambda}=0.1, t h_{\max }^{\lambda}=1, \alpha_{\lambda}=\pi /\left(t h_{\max }^{\lambda}-t h_{\min }^{\lambda}\right)$, and $\gamma_{\lambda}=-\alpha_{\lambda} t h_{\min }^{\lambda}$. This approach enables a smooth squeeze action, ranging from no squeeze when $\hat{\lambda}_{2}<t h_{\text {min }}^{\lambda}$ to the maximum squeeze when $\hat{\lambda}_{2} \geq t h_{\max }^{\lambda}$ (see [21] for additional details on the construction of $\Delta b_{p, c}$ ).

3) Subjects: Nine participants took part to the experiment, including two women and seven men (age $21-31$ ), all righthand dominant. The experimenter explained the procedures and spent about three minutes adjusting the setup to be comfortable. One practice trial was allowed.
4) Results: To evaluate the effectiveness of our teleoperation system and the usefulness of wearable haptic feedback, we evaluated (i) the completion time, (ii) the overall path length, (iii) the activation time of the squeezing action, and (iv) the perceived effectiveness of the different conditions.

Average completion time for conditions $\mathrm{V}$ and $\mathrm{CV}$ was $359.8 \mathrm{~s}$ and $296.6 \mathrm{~s}$, respectively (not shown in the figure). A paired-samples t-test determined that the task completion time did not differed statistically significantly between the conditions. Figure $8 \mathrm{a}$ shows the average trajectory length covered by the leader during the task. A paired-samples t-test determined that this metric differed statistically significantly between conditions $(t(8)=3.274, p=0.011, a=0.05)$. To evaluate the benefit of using the pressure/squeeze feedback, we also analyzed the percentage of time the squeezing action was activated (not shown in the figure). This metric indicates how often users commanded sudden increases of $\lambda_{2}^{d}$ (i.e., high values for $\hat{\lambda}_{2}^{d}$ ). In condition $\mathrm{V}$, when users did not wear the cutaneous device, this metric indicates the percentage of time the squeezing action would have been activated if the cutaneous device was worn. A paired-samples t-test determined that in condition CV users requested significantly lower values for $\hat{\lambda}_{2}^{d}$ than in condition $\mathrm{V}$, leading to a smoother behavior of the robots $(t(8)=3.019, p=$ $0.017, a=0.05)$. Immediately after the experiment, subjects were also asked to report the effectiveness of both feedback conditions in completing the given task using bipolar Likerttype nine-point scales. Fig. $8 \mathrm{~b}$ shows the perceived effectiveness. A Wilcoxon signed rank test showed a statistically significant difference between the means of the two feedback conditions $(z=2.527, p=0.012, a=0.05)$. Finally, all subjects but one found using wearable haptic feedback to be the most effective at completing the teleoperation task.

\section{DISCUSSION AND CONCLUSIONS}

This paper presents a novel wearable haptic device for the forearm and its evaluation in two robotic teleoperation scenarios. The device is able to provide skin stretch, pressure, and vibrotactile stimuli. Two servo motors actuate an elastic fabric belt wrapped around the arm. The belt also houses four vibrotactile motors, positioned evenly around the arm at 90 degrees from each other. We carried out a detailed perceptual characterization of the device as well as two human-subjects teleoperation experiments, enrolling a total of 34 subjects.

Section IV-A considers a shared-control teleoperation experiment with a virtual 6-DoF robotic manipulator. Haptic feedback provided information about the constraints imposed by the shared-control algorithm on the motion of the slave robot. We evaluated the performance of the system either providing haptic feedback with our device $(\mathrm{C})$ or no haptic feedback at all $(\mathrm{N})$. Using our wearable device improved the performance of the task in all the considered metrics, and the vast majority of the subjects found condition $\mathrm{C}$ more effective. The largest improvement is seen in the trajectory length metric, showing that haptic feedback enables users to follow more closely the constrained motion of the robot and avoid problematic situations (e.g., workspace and joints limits). The second largest improvement is registered for the perceived 
effectiveness metric, proving that even inexperienced users can appreciate and effectively use our device. The difference between the two conditions in the other metric is less marked, but still statistically significant at the considered significance level. Finally, three subjects complained that, in condition N, it was sometimes hard to match the motion of their hand to the one of the simulated robot. In fact, in condition $\mathrm{N}$, users have no direct information about the motion constraints of the slave robot. Therefore, the motion of the user's hand can significantly differ from the allowed motions of the slave robot, ending up in a very different and confusing configuration. This behavior was not registered in condition $\mathrm{C}$, since the haptic feedback kept the human users close to the slave's allowed directions of motion.

Section IV-B considers the teleoperation of a fleet of ten virtual quadrotors. We evaluated the performance of the system providing either visual and haptic feedback together (CV) or visual feedback alone $(\mathrm{V})$. Visual feedback consisted of a blue segment indicating on the screen the target direction of motion. Haptic feedback provided navigation information about the path to follow (vibrations) and the connectivity of the fleet (squeeze). Providing haptic feedback through our wearable device significantly improved the performance of the task in all the considered metrics but completion time. Seven subjects out of nine found condition $\mathrm{CV}$ more effective. The two subjects who preferred condition $\mathrm{V}$ did not like condition $\mathrm{CV}$ because their arm felt tired at the end of the experiment. Differently from Sec. IV-A, in this experiment we always provided navigation information through visual feedback (i.e., a segment pointing toward the target via-point). Haptic feedback then reinforced this information through vibrotactile stimuli provided by our device. All subjects reported that the task was easy to complete in both conditions. However, haptic feedback helped them in reaching faster the via-points. Results prove the viability and effectiveness of haptic feedback and of our device for robotic teleoperation applications. We should also consider that humans are used to follow visual navigation cues (e.g., turn-by-turn car navigation systems, road signs), while they are not used to follow haptic navigation stimuli at all. For this reason, we expect more training to significantly improve the performance of the haptic modality in all the considered applications.

In the next future, we will focus on reducing the form factor and weight of our device, with the objective of improving the comfort and wearability of the system. We also plan to study the role of skin elasticity in the actuation of our elastic belt, as well as how the perception of our haptic stimuli changes as the dimensions of the arm change. We also want to study the best way of fixing the device onto the forearm, with the objective of eliciting the most effective sensations. In this paper, we used a second stationary belt to fasten the upper static platform to the proximal side of the arm. In this respect, we are interested in exploring different, and potentially more effective, fasting approaches (e.g., directly clamping the upper structure onto the forearm). Finally, we will extend the evaluation of our device to real-world applications, starting from the teleoperation of a real robotic manipulator and quadrotors fleet, where we will also try to use all the three feedback modalities (skin stretch, pressure, vibrotactile) at the same time.

\section{REFERENCES}

[1] C. Pacchierotti, L. Meli, F. Chinello, M. Malvezzi, and D. Prattichizzo, "Cutaneous haptic feedback to ensure the stability of robotic teleoperation systems," International Journal of Robotics Research, vol. 34, no. 14, pp. 1773-1787, 2015.

[2] J. Bimbo, C. Pacchierotti, M. Aggravi, N. Tsagarakis, and D. Prattichizzo, "Teleoperation in cluttered environments using wearable haptic feedback," in Proc. IEEE/RSJ IROS, Vancouver, Canada, Sep. 2017, pp. 3401-3408.

[3] I. Bortone, D. Leonardis, M. Solazzi, C. Procopio, A. Crecchi, L. Briscese, P. Andre, L. Bonfiglio, and A. Frisoli, "Serious game and wearable haptic devices for neuro motor rehabilitation of children with cerebral palsy," in Converging Clinical and Engineering Research on Neurorehabilitation II. Springer, 2017, pp. 443-447.

[4] S. Scheggi, M. Aggravi, and D. Prattichizzo, "Cooperative Navigation for Mixed Human-Robot Teams Using Haptic Feedback," IEEE Trans. on Human-Machine Systems, vol. 47, no. 4, pp. 462-473, 2016.

[5] H. Culbertson, J. M. Walker, M. Raitor, and A. M. Okamura, "WAVES: A Wearable Asymmetric Vibration Excitation System for Presenting Three-Dimensional Translation and Rotation Cues," in Proc. Conf. on Human Factors in Computing Systems (CHI), 2017, pp. 4972-4982.

[6] M. Maisto, C. Pacchierotti, F. Chinello, G. Salvietti, A. De Luca, and D. Prattichizzo, "Evaluation of wearable haptic systems for the fingers in augmented reality applications," IEEE Trans. Haptics, vol. 10, no. 4, pp. 511-522, Oct 2017

[7] K. Bye, "Tricking the brain is the only way to achieve a total haptics solution," https://goo.gl/dvbRfu, 2017.

[8] S. Scheggi, L. Meli, C. Pacchierotti, and D. Prattichizzo, "Touch the virtual reality: using the leap motion controller for hand tracking and wearable tactile devices for immersive haptic rendering," in Proc. ACM SIGGRAPH, 2015, p. 31.

[9] C. Pacchierotti, A. Tirmizi, G. Bianchini, and D. Prattichizzo, "Enhancing the performance of passive teleoperation systems via cutaneous feedback," IEEE Trans. Haptics, vol. 8, no. 4, pp. 397-409, 2015.

[10] K. Bark, J. Wheeler, P. Shull, J. Savall, and M. Cutkosky, "Rotational skin stretch feedback: A wearable haptic display for motion," IEEE Trans. Haptics, vol. 3, no. 3, pp. 166-176, 2010.

[11] A. Stanley, K. J. Kuchenbecker et al., "Evaluation of tactile feedback methods for wrist rotation guidance," IEEE Trans. Haptics, vol. 5, no. 3, pp. 240-251, 2012.

[12] S. Casini, M. Morvidoni, M. Bianchi, M. Catalano, G. Grioli, and A. Bicchi, "Design and realization of the CUFF-clenching upperlimb force feedback wearable device for distributed mechano-tactile stimulation of normal and tangential skin forces," in Proc. IEEE/RSJ Int. Conf. on Intelligent Robots and Systems (IROS), 2015, pp. 1186-1193.

[13] M. Bianchi, "A fabric-based approach for wearable haptics," Electronics, vol. 5, no. 3, p. 44, 2016

[14] F. Chinello, C. Pacchierotti, N. G. Tsagarakis, and D. Prattichizzo, "Design of a wearable skin stretch cutaneous device for the upper limb," in Proc. IEEE Haptics Symposium (HAPTICS), 2016, pp. 14-20.

[15] C. Pacchierotti, S. Sinclair, M. Solazzi, A. Frisoli, V. Hayward, and D. Prattichizzo, "Wearable haptic systems for the fingertip and the hand: taxonomy, review, and perspectives," IEEE Trans. Haptics, vol. 10, no. 4, pp. 580-600, 2017.

[16] M. Aggravi, G. Salvietti, and D. Prattichizzo, "Haptic wrist guidance using vibrations for Human-Robot teams," in Proc. IEEE RO-MAN, 2016.

[17] C. Pacchierotti, G. Salvietti, I. Hussain, L. Meli, and D. Prattichizzo, "The hRing: a wearable haptic device to avoid occlusions in hand tracking," in Proc. IEEE Haptics Symposium (HAPTICS), 2016, pp. 134-139.

[18] F. Abi-Farraj, N. Pedemonte, and P. Robuffo Giordano, "A visualbased shared control architecture for remote telemanipulation," in Proc. IEEE/RSJ IROS, 2016, pp. 4266-4273.

[19] F. Chinello, C. Pacchierotti, J. Bimbo, N. G. Tsagarakis, and D. Prattichizzo, "Design and evaluation of a wearable skin stretch device for haptic guidance," IEEE Robotics and Automation Letters, Special Issue on Human Cooperative Wearable Robotic Systems, vol. 3, no. 1, pp. 524-531, 2018.

[20] D. Prattichizzo, C. Pacchierotti, and G. Rosati, "Cutaneous force feedback as a sensory subtraction technique in haptics," IEEE Trans. Haptics, vol. 5, no. 4, pp. 289-300, 2012

[21] P. Robuffo Giordano, A. Franchi, C. Secchi, and H. H. Bülthoff, "A passivity-based decentralized strategy for generalized connectivity maintenance," Int. J. Robotics Res, vol. 32, no. 3, pp. 299-323, 2013.

[22] C. Secchi, A. Franchi, H. H. Bülthoff, and P. Robuffo Giordano, "Bilateral Control of the Degree of Connectivity in Multiple Mobile-robot Teleoperation," in Proc. IEEE Int. Conf. on Robotics and Automation, 2013, pp. 3645-3652. 